\section{Mobility, Degradation, and Uptake of Indaziflam under Greenhouse Conditions}

\author{
Amir M. González-Delgado and Manoj K. Shukla \\ Plant and Environmental Sciences Department, New Mexico State \\ University, P.O. Box 30003, MSC 3Q, Skeen Hall Room N 127, Las \\ Cruces, NM 88003-8003
}

Additional index words. pecan, phytotoxicity, preferential flow, root uptake

\begin{abstract}
The objectives of this study were to evaluate the leaching, degradation, uptake, and mass balance of indaziflam, as well as its potential to produce phytotoxicity effects on young pecan trees. Pecan trees were planted in pots with homogeneous porous media (sandy loam soil), preferential flow channels open to the soil surface, and shallow tillage at the soil surface. Pots were treated with indaziflam at two application rates of 25 and 50 $\mathrm{g}$ a.i./ha in 2014 and 2015. Each pecan tree was irrigated with $7 \mathrm{~L}$ of water every 2 weeks during the growing season. An irrigation volume of $2 \mathrm{~L}$ was used to maximize indaziflam retention time in the soil from Dec. 2015 until the end of the trees' dormant stage. In 2014, leachate samples were collected after each irrigation for quantifying indaziflam mobility. Soil samples were collected at depths of 0 to 12 and 12 to $24 \mathrm{~cm}$ after 45,90 , and 135 days of indaziflam application, and leaf samples were collected at the end of the growing season to quantify mobility and uptake. Indaziflam was detected in leachate samples, and the leaf indaziflam content increased with increasing application rate. Indaziflam and its breakdown products were detected at both sampling depths. Mass recovery and half-life values for indaziflam in the soil ranged from $38 \%$ to $68 \%$ and 63 to 99 days, respectively. No phytotoxicity effects were observed from increasing application rate and retention time of indaziflam in the soil. Most of the applied indaziflam was retained in the soil at shallow depth.
\end{abstract}

Pecan [Carya illinoinensis (Wangenh.) K. Koch] is an important crop in southern New Mexico, and appropriate application of agrochemicals for pest control is required to maintain water quality and sustain pecan production. Indaziflam (Alion Herbicide; Bayer CropScience, Research Triangle Park, $\mathrm{NC}$ ) is a cellulose biosynthesis inhibitor registered in United States to be used for preemergence control of annual grass and broadleaf weeds (Alonso et al., 2011; Brabham et al., 2014). However, indaziflam is not approved to be used in the states of New Mexico and Arizona. In 2012, sporadic herbicide injury symptoms that included necrosis of leaves and varying trunk injuries were reported $\approx 3-4$ months after indaziflam application in two pecan orchards located in New Mexico and Arizona (González-Delgado

Received for publication 2 Feb. 2020. Accepted for publication 23 Apr. 2020.

Published online 25 June 2020.

This work was supported by grants from Bayer CropScience. We thank Bayer CropScience, Research Triangle Park, North Carolina, for providing analytical data of indaziflam. We also thank Nakayama Professorship, NIFA, New Mexico State University Agricultural Experiment Station, and Frank Sholedice for editing the manuscript.

A.M.G.-D. is the corresponding author. E-mail: amgonz4@nmsu.edu.

This is an open access article distributed under the CC BY-NC-ND license (https://creativecommons. org/licenses/by-nc-nd/4.0/). showed an intermediate lateral movement potential compared with other herbicides (pronamide $>$ simazine $\geq$ indaziflam $>$ amicarbazone $\geq$ dithiopyr $>$ prodiamine) after evaluating the influence of a major simulated storm event in a field experiment (Leon et al., 2016).

Information on the fate and transport behavior of indaziflam is required to minimize off-target plant injury risk (Jeffries et al., 2014). Previous studies reported a decrease of indaziflam injury to bermudagrass with increasing organic matter and clay contents under laboratory conditions (Jones et al., 2013a, 2013b; Schneider et al., 2015). A field study conducted in a New Mexico orchard using different indaziflam application rates reported that indaziflam moved outside the study site through lateral flow, and it was consistently detected in soil samples collected at 30- to 46- $\mathrm{cm}$ depth between 63 and $126 \mathrm{~d}$ after treatment (González-Delgado et al., 2016). Faster movement of solutes and water in the soil under field conditions is influenced by macropores that promote their preferential flow (Beven and Germann, 1982; Shipitalo et al., 2000). Preferential flow channels in the porous media could enhance herbicide movement from the soil surface to the root zone ultimately leading to herbicide injuries in crops (Carter, 2000; Feucht, 1988). The overall objective of this research was to evaluate various scenarios to understand injury to pecan trees due to indaziflam application in a greenhouse. However, to the best of our knowledge, no study has evaluated the indaziflam in leachate samples as well as uptake by plants. Therefore, specific objectives of this study were to evaluate the leaching, degradation, uptake, and mass balance of indaziflam due to 1) the presence of preferential flow channels, 2) application rates, and 3) an increase in resident time of indaziflam in soil water in a greenhouse experiment. The fourth objective was to quantify the mobility of indaziflam breakdown products in the soil.

\section{Materials and Methods}

its fate and transport under laboratory and field conditions (Guerra et al., 2014; Trigo et al., 2014).

Indaziflam breakdown products are indaziflam-triazine indanone, indaziflamcarboxylic acid, indaziflam-hydroxyethyl, indaziflam-olefin, and indaziflam-triazinediamine. Among these, indaziflam-triazine indanone, indaziflam-carboxylic acid, and indaziflamtriazinediamine are the main breakdown products and are more mobile than indaziflam in the soil (Alonso et al., 2016; Trigo et al., 2014). Previous studies reported that the leaching of indaziflam was positively influenced by increasing application rate and amount of rainfall in soil column experiments (Jhala et al., 2012a; Jhala and Singh, $2012 b$ ). Similarly, greater percent mass recoveries of indaziflam were found in a floodirrigated field treated with the application rate of 36.5 compared with $73.1 \mathrm{~g}$ a.i./ha (González-Delgado et al., 2016). Indaziflam
Experimental details. A greenhouse study was conducted from 18 Aug. 2014 to 5 May 2016, at New Mexico State University's Fabian Garcia Science Center in Las Cruces, NM. Two-year-old pecan trees of the variety 'Wichita' planted in pots were obtained from a local nursery (Archer Farms, Inc., Las Cruces, NM) and replanted in $0.035 \mathrm{~m}^{3}$ pots with an inner diameter and height of $36 \mathrm{~cm}$. Sandy loam soil (mixed, thermic Typic Torripsamment; Table 1) was used to match the soil texture $(76 \pm 0.9 \%$ sand and $8 \pm 0.5 \%$ clay) of typical New Mexico and Arizona orchards where sporadic herbicide injury symptoms were reported after indaziflam application in 2012 (González-Delgado et al., 2015).

Some of the possible causes of injury due to indaziflam related to soil water regimes were thought to be faster movement through sandier soils, preferential flow toward roots, 
Table 1. Average particle size distribution, bulk density, organic matter content, and soil texture of sandy loam soil used to plant 2-year-old pecan trees in pots.

\begin{tabular}{|c|c|c|c|c|c|}
\hline Sand (\%) & Silt $(\%)$ & Clay $(\%)$ & Bulk density $\left(\mathrm{g} \cdot \mathrm{cm}^{-3}\right)$ & $\mathrm{OM}(\%)^{\mathrm{z}}$ & Soil texture \\
\hline $76 \pm 0.9$ & $15 \pm 1.1$ & $8 \pm 0.5$ & $1.42 \pm 0.26$ & $1.5 \pm 0.2$ & Sandy loam \\
\hline
\end{tabular}

${ }^{\mathrm{z}}$ Soil organic matter content.

and higher resident time of the herbicide in the root zone. Therefore, three soil manipulations were created in 21 pots replicated thrice during 2014 and 2015 experiments. A set of nine trees was planted in pots with a homogeneous porous media $(\mathrm{H}$; no preferential flow channels), a second set of six trees with preferential flow (PF) channels open at the soil surface, and a third set of six trees with the combination of preferential flow channels underlying the shallowly tilled $(<5$ $\mathrm{cm})$ surface (ST). Preferential flow channels were created by placing four $0.64-\mathrm{cm}$ diameter wooden sticks at opposite sides of the pots during the planting process to promote root exposure to indaziflam (GonzálezDelgado et al., 2017).

A hand pump sprayer was used to spray indaziflam directly over the soil with two application rates of 25 (R1) and 50 (R2) g a.i./ ha on 18 Aug. 2014, 27 July 2015, and 22 Dec. 2015. The highest application rate (50 g a.i./ha) for this study is slightly lower than field application rate (73.1 g a.i./ha) considering the lower dissipation rate of herbicide in greenhouse than in the field. Each tree received an application volume of $300 \mathrm{~mL}$ that was distributed as $150 \mathrm{~mL}$ to each side of the pot to ensure an even indaziflam application over the soil surface. Limited contact between sprayed indaziflam and lower section of the trunk adjacent to the soil surface took place. Proper precautions were taken to ensure herbicide does not drift away from applied spot. Three pots with homogeneous porous media were used as a control and three replicates were used for each application rate and soil surface manipulation. Thus, there were 18 pots [ 2 (rate) $\times 3$ (soil condition) $\times 3$ (rep)] where herbicide was applied and three pots with no application (overall $=21$ ). Pots treated with rate $1(\mathrm{R} 1=25 \mathrm{~g}$ a.i./ha of indaziflam) were labeled as HR1, PFR1, and STR1, while those treated with rate 2 (R2 = $50 \mathrm{~g}$ a.i./ha) were labeled as HR2, PFR2, and STR2. A watering can was used to irrigate the pots with $7 \mathrm{~L}(69 \mathrm{~mm})$ of tap water $24 \mathrm{~h}$ after the indaziflam application and once every 2 weeks until the end of the growing season in 2014 and 2015. The volume of irrigation was close to the volume of irrigation water applied in the pecan orchard where injuries to trees were attributed to indaziflam application. The irrigation volume ensured exposure of roots to indaziflam due to water movement and ensured that enough water could be collected from drainage to quantify the leaching potential of indaziflam.

To increase the resident time of indaziflam in the soil and to maximize root exposure to indaziflam, between Dec. 2015 and May 2016, the pots were irrigated with only 2 $\mathrm{L}$ (19.6 mm) of water to ensure no leaching. Both application rates of indaziflam were applied to the pots on 22 Dec. 2015, when trees were dormant. In 2016, the mobility of indaziflam breakdown products was investigated in the soil. This experiment involved three new trees under control, and three each in PFR1 and PFR2 treatments.

Leachate, soil and leaf sampling. The leachate from the pots was collected in containers after irrigations on 19 Aug., 6 Sept., 24 Sept., 9 Oct., 23 Oct., 4 Nov., and 19 Nov. $2014(1,18,36,51,65,77$, and $92 \mathrm{~d}$ after indaziflam application). Water samples were collected from every treatment for indaziflam analysis using $30-\mathrm{mL}$ polystyrene-plastic vials (Fisher Scientific Company, LLC, Hampton, NH). Plastic vials were wrapped with plastic paraffin film (Fisher Scientific Company) to prevent contamination and accidental spill of water samples during the shipping process. No leachate samples were analyzed during 2015 and 2016.

Soil samples were collected from depths of 0 to 12 and 12 to $24 \mathrm{~cm}$ using a push probe and stored in Ziploc plastic bags. After collecting soil samples, the holes in the pots were filled with the sieved sandy loam soil. Samples were collected from pots close to 45 and $90 \mathrm{~d}$ (2 Oct. and $14 \mathrm{Nov}$.) after indaziflam application in 2014 based on an average halflife of $41 \mathrm{~d}$ derived from field dissipation studies conducted by Bayer CropScience (personal communication, 2015). During 2015, soil samples were collected 45, 90 , and $135 \mathrm{~d}$ after application (10 Sept., 27 Oct., and 10 Dec.).

Soil samples collected on 5 Feb., 21 Mar., and 5 May 2016 (45, 90, and $135 \mathrm{~d}$ after application) were analyzed for indaziflam as well as indaziflam breakdown products. The trees were visually evaluated for phytotoxicity effects during their dormant and active stages on a weekly basis. Ten leaf samples were collected from every treatment to quantify the uptake and translocation. Leaf samples were collected only on 19 Nov. 2014 and stored in Ziploc plastic bags just before dormancy.

All leachate, soil, and leaf samples were stored in a refrigerated room at $4{ }^{\circ} \mathrm{C}$. All samples were sent to Bayer CropScience for analysis. Indaziflam and its metabolites were extracted from the sample media by adding 80:20 (v/v) acetonitrile:water, followed by microwave extraction for $20 \mathrm{~min}$ at a maximum temperature of $60{ }^{\circ} \mathrm{C}$. After extraction, the mixture was fortified with isotopically (stable nitrogen-15) labeled internal standards of indaziflam $\{\mathrm{N}-[(1 \mathrm{R}, 2 \mathrm{~S})-2,3$-dihydro-2,6-dimethyl-1H-inden-1-yl]-6-[(1R)-1-fluoroethyl]-1, 3,5-triazine-2,4-diamine-- ${ }^{15} \mathrm{~N}_{4}$ \} and its metabolites and centrifuged. Samples were diluted before analysis with high performance liquid chromatography (HPLC) grade water. Indaziflam and its metabolites were analyzed by using a tandem mass spectrometer (LC/MS/ MS) (Sciex API 4000; AB Sciex LLC,
Framingham, MA) with a Synergy $4-\mu \mathrm{m}$ Fusion-RP HPLC column $(250 \times 2.0 \mathrm{~mm}$, $4 \mu \mathrm{m}, 80 \mathrm{~A}$ pore size) and flow rate of 0.2 $\mathrm{mL} / \mathrm{min}$. The quantification of indaziflam and its metabolites was based on the use of internal standards and comparison of peak areas to those of known standards. The trees were arranged using a completely randomized design with replications. One-way analysis of variance was performed to determine the significant differences among treatments at $95 \%$ probability level using SAS 9.2 (SAS Institute Inc., Cary, NC).

Mass balance of indaziflam. On 19 Aug. 2014, soil samples were collected about at 2$\mathrm{cm}$ depth from pots after indaziflam application and before the first irrigation event to determine the initial indaziflam concentration. Initially applied concentrations and residual concentrations in leachate and soil (total concentration from 0 to $24 \mathrm{~cm}$ depth) samples after $90 \mathrm{~d}$ of application were used to calculate the mass balance. Indaziflam concentrations in leaf samples were not included in the mass balance. The mass of indaziflam in the leachate and soil was calculated by multiplying the concentration by the total volume (L) of leachate and average soil mass $(35 \pm 6.4 \mathrm{~kg})$, respectively. Indaziflam masses in soil and leachate samples $90 \mathrm{~d}$ after application were divided by the initial applied mass to calculate the mass recovery.

\section{Results and Discussion}

Leaching of indaziflam. In 2014, indaziflam was detected in all leachate samples collected from every treatment during all seven irrigation events. No indaziflam was detected in leachate samples collected from controls. Generally, concentrations in leachate were higher in pots treated with $50 \mathrm{~g}$ a.i./ ha than $25 \mathrm{~g}$ a.i./ha during the first four sampling events and showed a decreasing trend with increasing time since application (Fig. 1). Higher concentrations of other herbicides, namely oxyfluorfen and atrazine, at greater depths were also reported at higher application rates (Gan et al., 1996; Horowitz and Elmore, 1991). Jhala et al. (2012a) also reported more indaziflam leaching with higher application rate. Greater leaching of indaziflam was observed in STR2 followed by PFR2, HR2, PFR1, HR1, and STR1. However, there was not a significant difference between the concentrations of the leachate samples collected from every treatment in 2014.

Indaziflam retention in soil. Indaziflam was detected in soil from every treated pot at depths of 0 to 12 and 12 to $24 \mathrm{~cm} 45$ and $90 \mathrm{~d}$ after the first application in 2014 (Fig. 2A and B). Soil indaziflam concentrations were significantly higher at depths of 0 to $12 \mathrm{~cm}$ than 12 to $24 \mathrm{~cm}$ after $45(P<0.0028)$ and $90(P<$ 
0.0018) days in 2014. This confirmed that most of the applied indaziflam remained within the surface soil across all treatments. No indaziflam in soil was detected in the

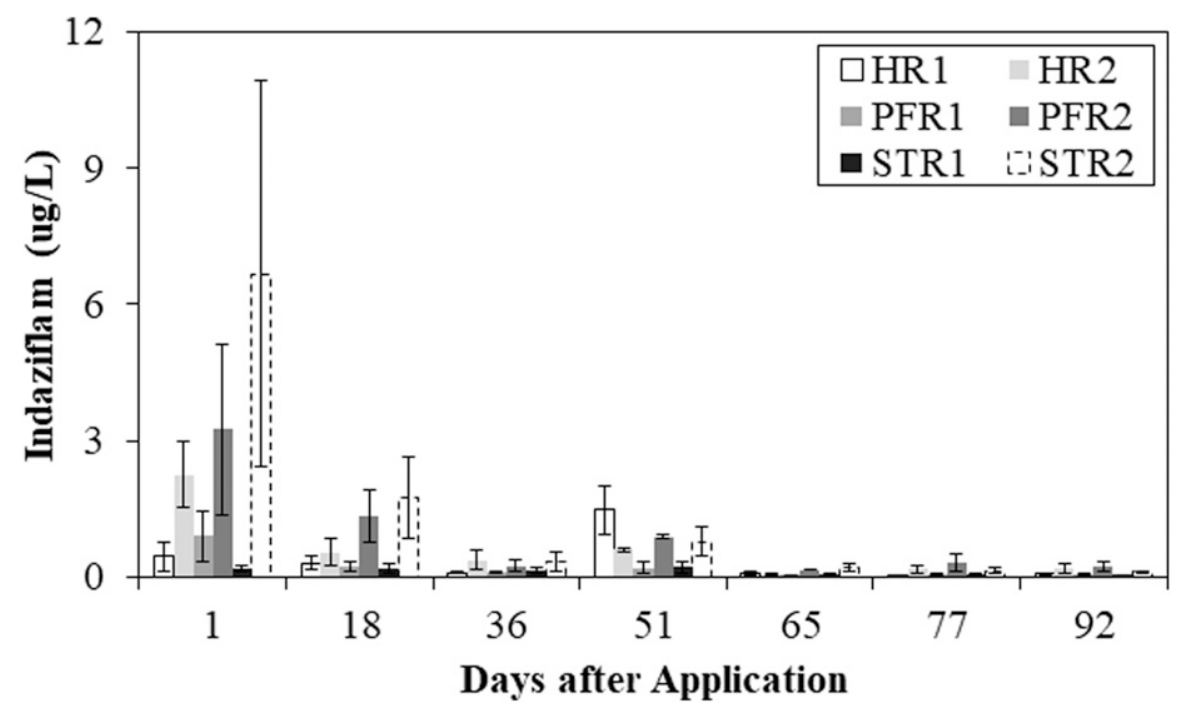

Fig. 1. Concentration of indaziflam in leachate samples collected from pots with homogeneous soil without preferential flow channels $(\mathrm{H})$, preferential flow channels open to the soil surface (PF), and preferential flow channels closed to the soil surface (ST) after being treated with 25 (rate $1=\mathrm{R} 1$ ) and 50 (rate $2=$ R2) g a.i./ha of indaziflam. Error bars represent standard error. between 45 and $90 \mathrm{~d}$ after application. The lack of significant difference in soil concentrations collected 45 and $90 \mathrm{~d}$ after application suggests that the degradation of indaziflam was slow under greenhouse conditions. The relative comparison between indaziflam concentrations in the leachate and soil samples collected from pots treated with 25 and $50 \mathrm{~g}$ a.i./ha showed that 42 and $59 \%$ of the applied indaziflam remained in the soil, respectively $90 \mathrm{~d}$ after application. Less than $1 \%$ of the applied indaziflam was found in the leachate collected from pots treated with both application rates. The use of plastic vials could have led to some loss of indaziflam in leachate samples due to adsorption that could have been minimized by using glass vials. The loss of indaziflam was expected to be low since indaziflam has an octanol-water partition coefficient $\left(\log \mathrm{K}_{\mathrm{ow}}\right)$ value of 2.8 which is similar to the $\log \mathrm{K}_{\mathrm{ow}}$ value of atrazine (2.6) and lower than Sethyl-dipropylthiocarbamate (3.2) and Oxyfluorfen (4.73) (Alonso et al., 2011; Patakioutas and Albanis, 2002; U.S. Environmental Protection Agency, 2007). Studies have reported that the sorption of herbicide is positively correlated with organic matter content that generally decreases with increasing depth (Alonso et al., 2011;
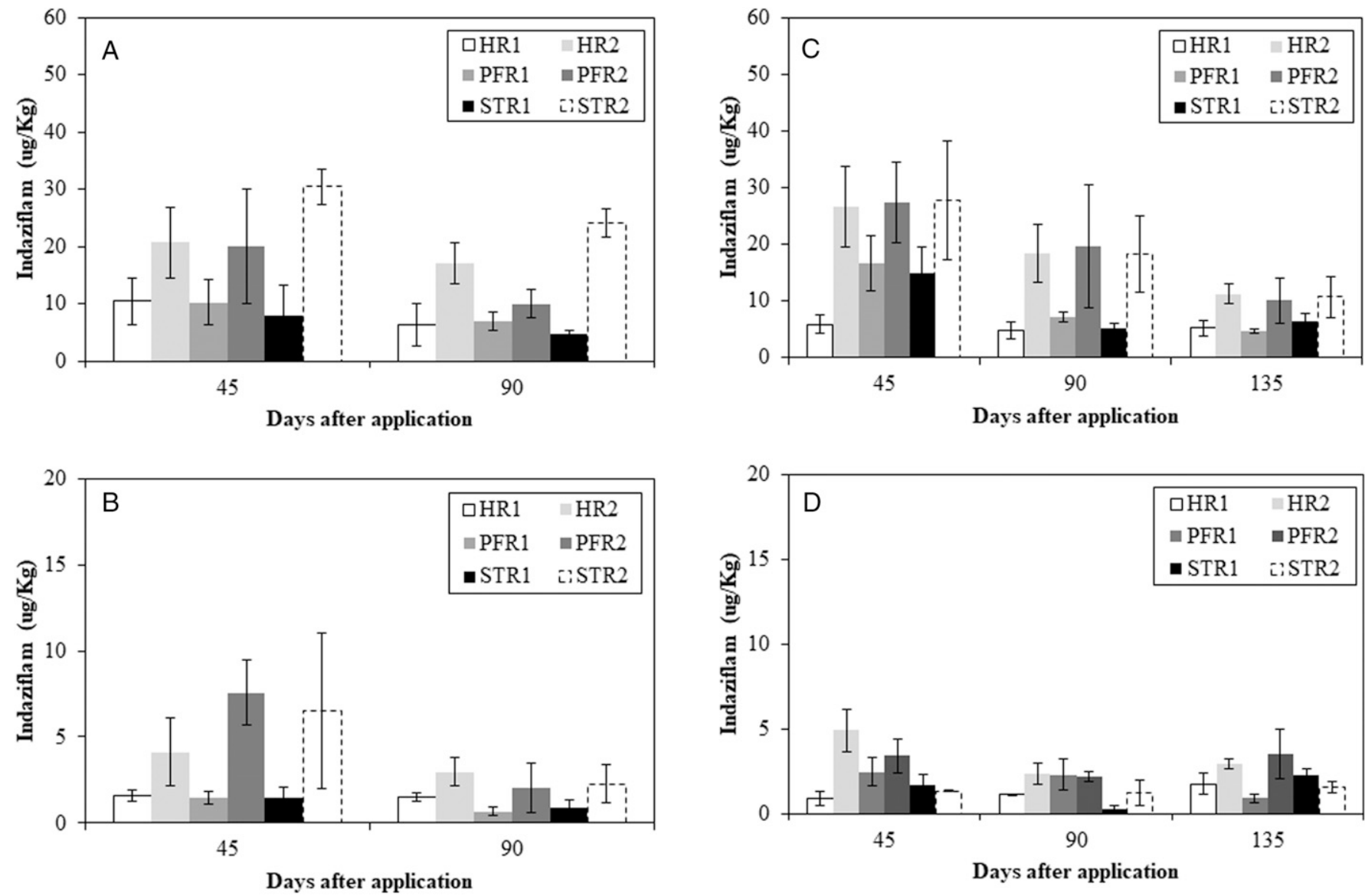

Fig. 2. Concentration of indaziflam in soil samples collected at (A) 0-12 and (B) 12-24 cm depths 45 and $90 \mathrm{~d}$ after treatment in 2014, and concentration of indaziflam in soil samples collected at (C) 0-12 and (D) 12-24 cm depths 45, 90, and $135 \mathrm{~d}$ after treatment in 2015 in pots with homogeneous soil without preferential flow channels $(\mathrm{H})$, preferential flow channels open to the soil surface (PF), and preferential flow channels closed to the soil surface (ST) using 25 (rate $1=\mathrm{R} 1$ ) and 50 (rate $2=\mathrm{R} 2$ ) $\mathrm{g}$ a.i./ha of indaziflam. Error bars represent standard error. 


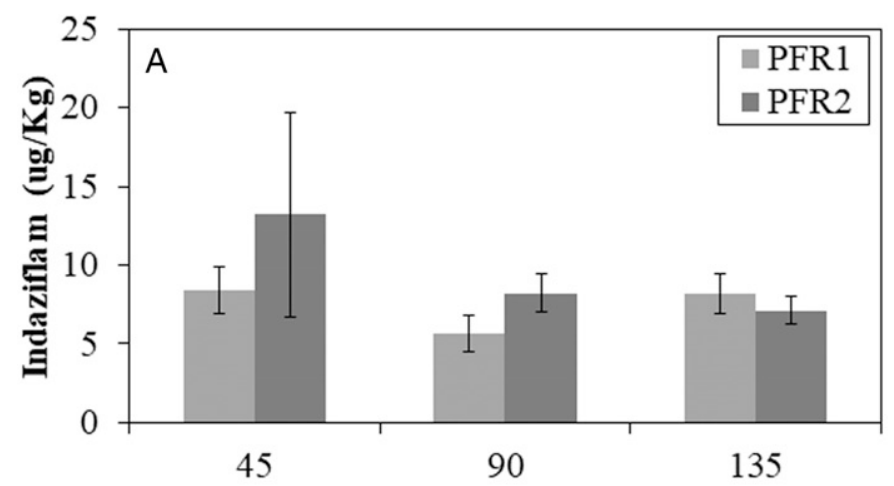

Days after application

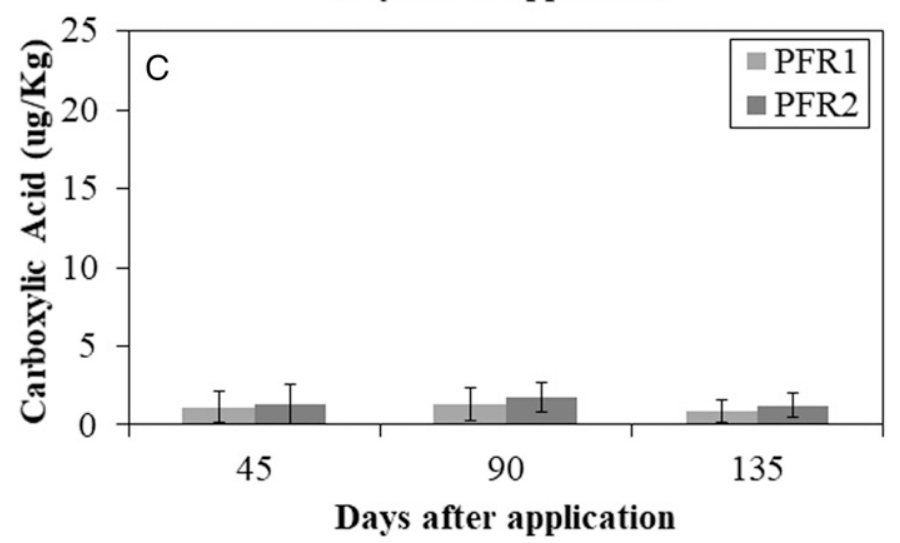

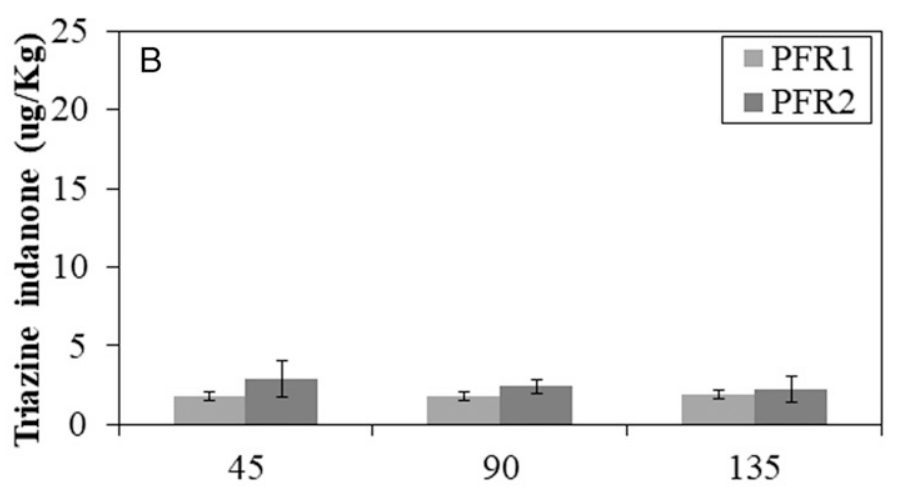

Days after application

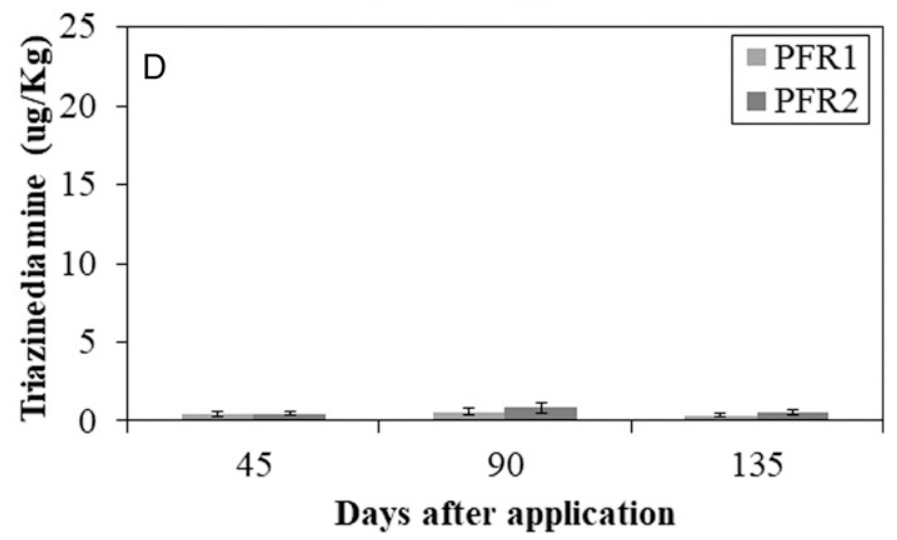

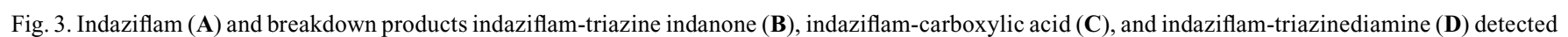
in soil samples collected at $0-24 \mathrm{~cm}$ depth in pots with preferential flow channels open to the soil surface (PF) 45,90 , and $135 \mathrm{~d}$ after being treated with 25 (rate $1=\mathrm{R} 1$ ) and 50 (rate $2=\mathrm{R} 2$ ) $\mathrm{g}$ a.i./ha of indaziflam. Error bars represent standard error.

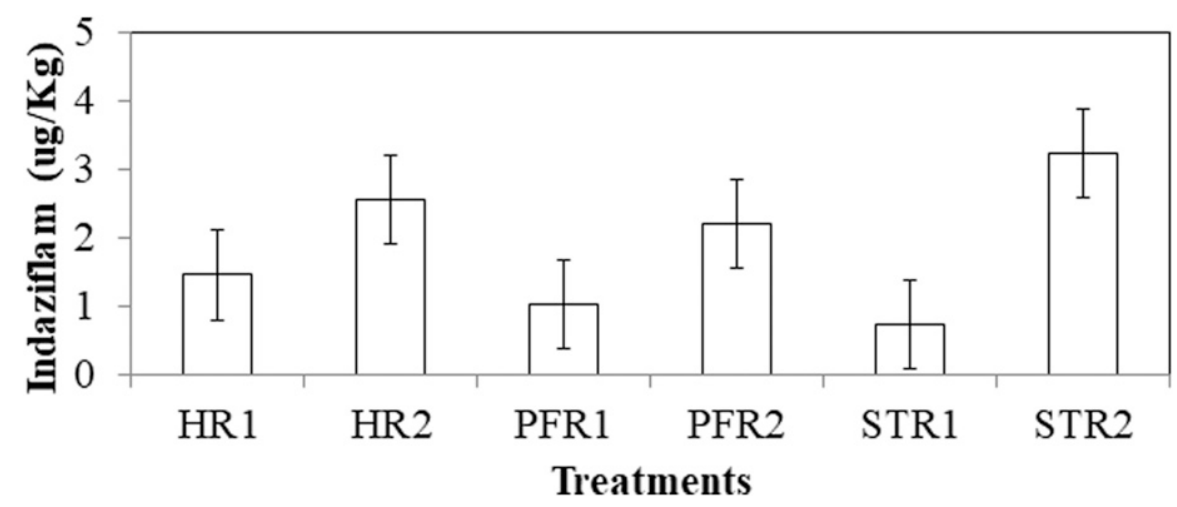

Fig. 4. Concentration of indaziflam in leaf samples collected from pots with homogeneous soil without preferential flow channels $(\mathrm{H})$, preferential flow channels open to the soil surface $(\mathrm{PF})$, and preferential flow channels closed to the soil surface (ST) after being treated with 25 (rate $1=\mathrm{R} 1$ ) and 50 (rate $2=$ R2) g a.i./ha of indaziflam in 2014. Error bars represent standard error.

Barriuso et al., 1992; González-Delgado et al., 2016; Rice et al., 2002). However, greater indaziflam concentrations at 0 - to $12-\mathrm{cm}$ depth compared with those at $12-$ to $24-\mathrm{cm}$ depth and leachate were not influenced by the distribution of organic matter in the soil. Pots were repacked with a sandy loam soil containing low organic matter content $(1.20 \%)$ that was homogenized after being sieved; therefore, there was no gradient in soil organic matter within the pots.

Indaziflam concentrations were higher in pots treated with the higher rate (50 g a.i./ha) than those in pots treated with the lower rate ( 25 g a.i./ha) (Fig. 2A and B). In contrast, a previous field study reported that indaziflam concentrations were generally greater in plots treated with a lower application rate of indaziflam (González-Delgado et al., 2016). This could be the result of enhanced microbial activity and vertical and lateral movement of indaziflam in the field. Under field conditions, a continuous nutrient supply through root exudation in the rhizosphere would promote more resilient and denser microbial populations capable of degrading herbicides (Roger et al., 1994).
Conflicting accounts are available in literature on microbial activity vs. applied concentration of herbicides. Gan et al. (1996) reported an increase of microbial activity at higher concentrations after evaluating the effect of concentration on the degradation of atrazine under field and laboratory conditions. Conversely, Fogg and Boxall (2003) reported that microbial activity was suppressed at concentrations exceeding the recommended application rates for isoproturon and chlorothalonil. Unlike field conditions, the movement of indaziflam through lateral flow was absent and restricted to vertical flow in pots. The STR2 treatment showed the highest soil concentration, followed by PFR2 and HR2 at 0- to $12-\mathrm{cm}$ depth $45 \mathrm{~d}$ after indaziflam application in 2014. However, there was no significant difference between them, indicating that the different soil surface treatments had no significant effect on indaziflam movement in sandy loam soil.

In 2015, indaziflam was consistently detected 45,90 , and $135 \mathrm{~d}$ after application at 0 to 12 -and 12- to 24-cm depths (Fig. 2C and D). Similar to 2014 , soil indaziflam concentrations were greater at 0 - to $12-\mathrm{cm}$ depth than at 12- to 24-cm depth for all treatments. Soil concentrations at both depths were not significantly different between treatments; however, concentrations at $0-$ to $12-\mathrm{cm}$ and $12-$ to $24-\mathrm{cm}$ depths were significantly different 45, 90, and $135 \mathrm{~d}$ after application $(P<$ $0.001)$. 
Table 2. Concentration and total mass of indaziflam in volume of leachate samples collected from pots with homogeneous soil without preferential flow channels $(\mathrm{H})$, preferential flow channels open to the soil surface $(\mathrm{PF})$, and preferential flow channels closed to the soil surface $(\mathrm{ST})$ after being treated with 25 (rate $1=$ R1) and 50 (rate $2=\mathrm{R} 2$ ) g a.i./ha of indaziflam in 2014.

\begin{tabular}{|c|c|c|c|c|c|c|c|c|c|c|c|c|c|c|c|}
\hline \multirow[b]{3}{*}{ Sample } & \multicolumn{7}{|c|}{ Indaziflam $(\mu \mathrm{g} / \mathrm{L})$} & \multicolumn{7}{|c|}{ Leachate (L) } & \multirow[b]{3}{*}{ Total mass in leachate $(\mathrm{mg})$} \\
\hline & \multicolumn{14}{|c|}{ Days after treatment } & \\
\hline & 1 & 18 & 36 & 51 & 65 & 77 & 92 & 1 & 18 & 36 & 51 & 65 & 77 & 92 & \\
\hline$\overline{\mathrm{HR} 1}$ & 0.43 & 0.29 & 0.08 & 1.46 & 0.09 & 0.03 & 0.06 & 0.92 & 0.75 & 1.79 & 0.47 & 0.54 & 0.94 & 0.31 & $1.55 \mathrm{E}-03$ \\
\hline HR2 & 2.25 & 0.53 & 0.35 & 0.59 & 0.06 & 0.16 & 0.18 & 1.96 & 1.70 & 2.63 & 1.05 & 1.11 & 1.49 & 1.20 & 7.35E-03 \\
\hline PFR1 & 0.88 & 0.21 & 0.08 & 0.19 & 0.03 & 0.05 & 0.06 & 1.38 & 1.51 & 1.21 & 1.22 & 0.71 & 1.21 & 1.08 & $2.01 \mathrm{E}-03$ \\
\hline PFR2 & 3.24 & 1.33 & 0.23 & 0.88 & 0.14 & 0.31 & 0.21 & 1.42 & 1.23 & 1.31 & 0.75 & 0.61 & 0.87 & 0.91 & $7.76 \mathrm{E}-03$ \\
\hline STR1 & 0.18 & 0.17 & 0.11 & 0.21 & 0.04 & 0.05 & 0.03 & 0.89 & 1.06 & 1.03 & 0.26 & 0.28 & 0.47 & 0.43 & $5.62 \mathrm{E}-04$ \\
\hline STR2 & 6.67 & 1.74 & 0.33 & 0.77 & 0.20 & 0.13 & 0.10 & 1.32 & 0.67 & 1.63 & 0.71 & 0.31 & 0.60 & 0.83 & $1.13 \mathrm{E}-02$ \\
\hline
\end{tabular}

Table 3. Indaziflam soil concentration and percent mass recoveries in soil and leachate samples collected from pots with homogeneous soil without preferential flow channels $(\mathrm{H})$, preferential flow channels open to the soil surface (PF), and preferential flow channels closed to the soil surface (ST) after being treated with 25 (rate $1=\mathrm{R} 1$ ) and 50 (rate $2=\mathrm{R} 2$ ) $\mathrm{g}$ a.i./ha of indaziflam in 2014 .

\begin{tabular}{lcccccc}
\hline & & \multicolumn{5}{c}{ Mass $(\mathrm{mg})$} \\
\cline { 3 - 6 } Sample & Indaziflam $(\mathrm{ug} / \mathrm{kg})$ & $\mathrm{M}_{\text {soil }}$ & $\mathrm{M}_{\text {leachate }}$ & $\mathrm{M}_{\text {total }}$ & $\mathrm{M}_{\text {initial }}$ & $\mathrm{M}_{\text {recovered }}(\%)$ \\
\hline HR1 & 7.77 & 0.27 & $1.55 \mathrm{E}-03$ & 0.27 & 0.60 & 46 \\
HR2 & 24.84 & 0.87 & $7.35 \mathrm{E}-03$ & 0.88 & 1.40 & 63 \\
PFR1 & 7.59 & 0.27 & $2.01 \mathrm{E}-03$ & 0.27 & 0.60 & 45 \\
PFR2 & 17.59 & 0.62 & $7.76 \mathrm{E}-03$ & 0.62 & 1.30 & 48 \\
STR1 & 6.10 & 0.21 & $5.62 \mathrm{E}-04$ & 0.21 & 0.60 & 38 \\
STR2 & 26.40 & 0.92 & $1.13 \mathrm{E}-02$ & 0.94 & 1.38 & 68 \\
\hline
\end{tabular}

$\mathrm{M}_{\text {soil }}=$ mass of indaziflam in soil; $\mathrm{M}_{\text {leachate }}=$ mass of indaziflam in leachate; $\mathrm{M}_{\mathrm{total}}=$ total indaziflam mass from soil and leachate; $M_{\text {initial }}=$ mass of indaziflam added to the pots; $M_{\text {recovered }}=$ percentage of indaziflam mass recovery $=\left[\left(\mathrm{M}_{\text {soil }}+\mathrm{M}_{\text {leachate }}\right) / \mathrm{M}_{\text {initial }}\right] \times 100$

Table 4. Indaziflam degradation coefficient and half-life values in soil samples collected from pots with homogeneous soil without preferential flow channels $(\mathrm{H})$, preferential flow channels open to the soil surface (PF), and preferential flow channels closed to the soil surface (ST) after being treated with 25 (rate $1=\mathrm{R} 1$ ) and 50 (rate $2=\mathrm{R} 2$ ) $\mathrm{g}$ a.i./ha of indaziflam in 2014.

\begin{tabular}{lccc}
\hline Treatment & $\mathrm{k}\left(\right.$ day $\left.^{-1}\right)$ & $\mathrm{t}_{1 / 2}($ day $)$ & $R^{2}$ \\
\hline HR1 & 0.009 & 77 & 0.99 \\
PFR1 & 0.009 & 77 & 0.99 \\
STR1 & 0.011 & 63 & 0.98 \\
HR2 & 0.007 & 99 & 0.95 \\
PFR2 & 0.008 & 87 & 0.95 \\
STR2 & 0.007 & 99 & 0.99 \\
\hline
\end{tabular}

Mobility of indaziflam breakdown products. Because there was no significant difference among soil surface treatments, only PFR1 and PFR2 treatments were selected to conduct the third experiment on determining the mobility of indaziflam breakdown products from late Dec. 2015 to 5 May 2016. Indaziflam, indaziflam-triazine indanone, indaziflam-carboxylic acid, and indaziflam-triazinediamine were detected 45,90 , and $135 \mathrm{~d}$ after application at both sampling depths and treatments, but indaziflam-hydroxyethyl and indaziflamolefin were not detected (Fig. 3). Similar to the results of 2014 and 2015 experiments, concentrations of indaziflam and its breakdown products were higher at 0 - to $12-\mathrm{cm}$ depth than at 12- to 24-cm depth. Generally, concentrations of indaziflam and indaziflam breakdown products in soil were numerically, but not statistically, greater in PFR2 than in PFR1 treatment. Indaziflam did not completely degrade $135 \mathrm{~d}$ after the applica- tion, probably due to lower dissipation factors present under greenhouse conditions compared with those present in the field. Relatively, concentrations of breakdown products of indaziflam in soil decreased in the following order: indaziflam-triazine indanone, indaziflam-carboxylic acid, and indaziflam-triazinediamine.

Currently, there is limited published information on the mobility and phytotoxicity of indaziflam breakdown products. Limited studies have shown that indaziflam breakdown products are more mobile than indaziflam (Trigo et al., 2014). Alonso et al. (2016) reported that the sorption order for indaziflam breakdown products was indaziflam-triazine indanone $>$ indaziflam-carboxylic acid > indaziflam-triazinediamine in six Brazilian Oxisols, while the sorption order in three United States Mollisols was indaziflamtriazine indanone $>$ indaziflam-carboxylic acid = indaziflam-triazinediamine in laboratory batch experiments. At the end of this greenhouse study, no phytotoxicity effects on young pecan trees were observed when indaziflam and its breakdown products where present in the soil. Similar results were reported by Grey et al. (2018) after exposing young pecan trees to multiple applications of indaziflam. However, studies on the mobility, degradation, and toxicity of indaziflam and indaziflam breakdown products should be conducted under different scenarios because there is limited published information available.

Indaziflam uptake. Indaziflam was detected in leaf samples collected at the end of the growing season during Dec. 2014. This showed that indaziflam was taken up by roots and transported from the roots to the leaves after treating the pots with 50 and $25 \mathrm{~g}$ a.i./ha of indaziflam (Fig. 4). Indaziflam was detected in leaf samples from every treatment except the control. However, there was no significant difference in leaf indaziflam concentrations across treatments. Devine et al. (1987) and Sterling et al. (1990) reported that root and shoot uptake of herbicides increased linearly with applied herbicide concentration after evaluating the effect under different application rates ( 1 to $10^{3} \mu \mathrm{mol}$ of chlorsulfuron and clopyralid and 0.1 to $100 \mu \mathrm{mol}$ of bentazon). Leaf samples were not collected in 2015 and 2016 because no phytotoxicity effects were observed in trees during these periods. Also, control and treated trees showed no significant difference in trunk diameter $(26 \pm 0.72$ and $27 \pm 2.3 \mathrm{~mm}$; respectively) and height (146 \pm 4 and $137 \pm 6$ $\mathrm{cm}$; respectively) at the end of the study. The absence of phytotoxicity effects in trees treated with indaziflam could not explain the sporadic herbicide injury symptoms reported in the New Mexico and Arizona orchards.

Mass balance of indaziflam. As expected, lower initial indaziflam concentrations were measured in the soil of HR1 $(17.48 \mathrm{ug} / \mathrm{kg})$, PFR1 (16.62 ug/kg), and STR1 (15.92 ug/kg) compared with those in HR2 $(39.01 \mathrm{ug} / \mathrm{kg})$, PFR2 (36.29 ug/kg), and STR2 (48.6 ug/kg). Initial indaziflam masses in the soil of HR1, PFR1, and STR1 ranged from 0.56 to $0.61 \mathrm{mg}$ and 1.27 to $1.70 \mathrm{mg}$ in HR2, PFR2, and STR2. Total mass of indaziflam in the leachate collected from the treatments ranged from $5.62 \times 10^{-4}$ to $1.13 \times 10^{-2} \mathrm{mg}$ at the end of the study (Table 2). The mass balance confirms that most of the mass recovery of indaziflam took place in the soil, followed by the leachate samples (Table 3 ). Percent mass recovery in pots treated with 25 and $50 \mathrm{~g}$ a.i./ha ranged from $38 \%$ to $46 \%$ and $48 \%$ to $68 \%$, respectively. About half of the applied indaziflam concentration was recovered in most of the treatments, except for STR1 that had the lowest mass recovery.

Indaziflam is not reported to be volatile in the environment, however its dissipation is dominated by the degradation and leaching processes (U.S. Environmental Protection Agency, 2010). Because most of the applied indaziflam was recovered in the soil, the loss of indaziflam during the experiment took place through the degradation process. Indaziflam half-lives in soil treated with 25 and 50 g a.i./ha ranged from 63 to 77 and 87 to $99 \mathrm{~d}$, respectively (Table 4). Half-life values were 
greater compared with the average half-life of $41 \mathrm{~d}$ used as reference; however, most of them are within the half-life range of 30 to $86 \mathrm{~d}$ derived from an earlier field study (González-Delgado et al., 2016). Lower half-life values from field studies than those from laboratory studies could be the result of greater degradation in the rhizosphere under field conditions (Roger et al., 1994; Shaner and Henry, 2007).

\section{Conclusion}

In this greenhouse pot study, indaziflam was slightly mobile. Concentrations in soil, leachate, and leaf samples increased with increasing application rates. Most of the mass recovery of indaziflam took place in the soil and ranged from $38 \%$ to $68 \%$, and half-life values in soil ranged from 63 to $99 \mathrm{~d}$. Indaziflam's breakdown products were also detected in soil samples 45, 90, and $135 \mathrm{~d}$ after application. Indaziflam showed the highest soil concentration, followed by indaziflamtriazine indanone, indaziflam-carboxylic acid, and indaziflam-triazinediamine. No phytotoxicity effects on pecan trees were observed after exposing their roots to the application rates of 25 and $50 \mathrm{~g}$ a.i./ha under various experimental soil conditions. No phytotoxicity effects were observed when the retention time of indaziflam and its breakdown products in the soil was increased. Indaziflam and its breakdown products were mobile as well as taken up by plants; therefore, soil and leaf measurements should be made periodically. Most of the applied indaziflam was retained in the soil at shallow depth.

\section{Literature Cited}

Alonso, D.G., W.C. Koskinen, R.S. Oliveira, Jr., J. Constantin, and S. Mislankar. 2011. Sorptiondesorption of indaziflam in selected agricultural soils. J. Agr. Food Chem. 59(24):1309613101.

Alonso, D.G., R.S. Oliveira, W.C. Koskinen, K.E. Hall, J. Constantina, and S. Mislankard. 2016. Sorption and desorption of indaziflam degradates in several agricultural soils. Sci. Agr. 73(2):169-176.

Barriuso, E., Ch. Fellerb, R. Calvet, and C. Cenic. 1992. Sorption of atrazine, terbutryn and 2,4-D herbicides in two brazilian oxisols. Geoderma 53(1-2):155-167.

Beven, K. and P. Germann. 1982. Macropores and water flow in soils. Water Resour. Res 18:1311-1325.
Brabham, C., L. Lei, Y. Gu, J. Stork, M. Barrett, and S. DeBolt. 2014. Indaziflam herbicidal action: A potent cellulose biosynthesis inhibitor. Plant Physiol. 166(3):1177-1185.

Carter, A.D. 2000. Herbicide movement in soils: Principles, pathways and processes. Weed Res. 40(1):113-122.

Devine, M.D., H.D. Bestman, and W.H. Vanden Born. 1987. Uptake and accumulation of the herbicides chlorsulfuron and clopyralid in excised pea root tissue. Plant Physiol. 85(1):8286.

Feucht, J.R. 1988. Herbicide injuries to treessymptoms and solutions. J. Arboric. 14(9):215219.

Fogg, P. and A.B.A. Boxall. 2003. Degradation of pesticides in biobeds: The effect of concentration and pesticide mixtures. J. Agr. Food Chem. 51(18):5344-5349.

Gan, J., R.L. Becker, W.C. Koskinen, and D.D. Buhler. 1996. Degradation of atrazine in two soils as a function of concentration. J. Environ. Qual. 25(5):1064-1072.

González-Delgado, A.M., J. Ashigh, M.K. Shukla, and R. Perkins. 2015. Mobility of indaziflam influenced by soil properties in a semi-arid area. PLoS ONE 10(5):e0126100.

González-Delgado, A.M., J. Ashigh, M.K. Shukla, and R. Perkins. 2016. Effect of application rate and irrigation on the movement and dissipation of indaziflam. J. Environ. Sci. 51:111-119.

González-Delgado, A.M., M.K. Shukla, and B. Schutte. 2017. Effect of indaziflam application and soil manipulations on pecan evapotranspiration and gas exchange parameters. HortScience 52:1-6

Grey, T.L., K. Rucker, L. Wells, and X. Luo. 2018. Response of young pecan trees to repeated applications of indaziflam and halosulfuron. HortScience 53:313-317.

Guerra, N., A.M. Oliveira Neto, R.S. Oliveira Jr, J. Constantin, and H.K. Takano. 2014. Sensibility of plant species to herbicides aminocyclopyrachlor and indaziflam. Planta Daninha 32(3):609-617.

Horowitz, M. and M.C. Elmore. 1991. Leaching of oxyfluorfen in container media. Weed Technol. 5(1):175-180

Jeffries, M.D., D.J. Mahoney, and T.W. Gannon. 2014. Effect of simulated indaziflam drift rates on various plant species. Weed Technol. 28(4):608-616.

Jhala, A.J., A.H.M. Ramirez, and M. Singh. 2012a. Leaching of indaziflam applied at two rates under different rainfall situations in florida candler soil. Bull. Environ. Contam. Toxicol. 88(3):326-332.

Jhala, A.J. and M. Singh. 2012b. Leaching of indaziflam compared with residual herbicides commonly used in florida citrus. Weed Technol. 26(3):602-607.
Jones, P.A., J.T. Brosnan, D.A. Kopsell, and G.K Breeden. 2013a. Effect of reed-sedge peat moss on hybrid bermudagrass injury with indaziflam and prodiamine in sand-based root zones. Weed Technol. 27(3):547-551.

Jones, P.A., J.T. Brosnan, D.A. Kopsell, and G.K. Breeden. 2013b. Soil type and rooting depth affect hybrid bermudagrass injury with preemergence herbicides. Crop Sci. 53(2):660665.

Leon, R.G., J.B. Unruh, and B.J. Brecke. 2016. Relative lateral movement in surface soil of amicarbazone and indaziflam compared with other preemergence herbicides for turfgrass. Weed Technol. 30(1):229-237.

Patakioutas, G. and T.A. Albanis. 2002. Adsorption-desorption studies of alachlor, metolachlor, EPTC, chlorothalonil and pirimiphos-methyl in contrasting soils. Pest Manag. Sci. 58:352-362.

Rice, P.J., T.A. Anderson, and J.R. Coats. 2002. Degradation and persistence of metolachlor in soil: Effects of concentration, soil moisture, soil depth, and sterilization. Environ. Toxicol. Chem. 21(12):2640-2648.

Roger, P.A., I. Simpson, R. Oficial, S. Ardales, and R. Jimenez. 1994. Effects of pesticides on soil and water microflora and mesofauna in wetland rice fields: A summary of current knowledge and extrapolation to temperate environments. Aust. J. Exp. Agr. 34(7):1057-1068.

Schneider, J.G., J.B. Haguewood, E. Song, X. Pan, J.M. Rutledge, B.J. Monke, D.F. Myers, S.H Anderson, M.R. Ellersieck, and X. Xiong. 2015. Indaziflam effect on bermudagrass ( $C y n$ odondactylon L. Pers.) shoot growth and root initiation as influenced by soil texture and organic matter. Crop Sci. 55(1):429-436.

Shaner, L. and W.B. Henry. 2007. Field history and dissipation of atrazine and metolachlor in Colorado. J. Environ. Qual. 36(1):128-134.

Shipitalo, M.J., W.A. Dick, and W.M. Edwards. 2000. Conservation tillage and macropore factors that affect water movement and the fate of chemicals. Soil Tillage Res. 53:167-183.

Sterling, T.M., N.E. Balke, and D.S. Silverman. 1990. Uptake and accumulation of the herbicide bentazon by cultured plant cells. Plant Physiol. 92(4):1121-1127.

Trigo, C., W.C. Koskinen, and R.S. Kookana. 2014. Sorption-desorption of indaziflam and its three metabolites in sandy soils. J. Environ. Sci. Health B 49(11):836-843.

U.S. Environmental Protection Agency. 2007. Technical appendix b: Physicochemical properties for tri chemicals and chemical categories. 28 Apr. 2020. <https://www.epa.gov/sites/production/files/2014-03/documents/tech_app_b_ v215.pdf $>$.

U.S. Environmental Protection Agency. 2010. Pesticide fact sheet. Conditional registration. p. 1-108. 